\title{
Canceling the Gravity Gradient Phase Shift in Atom Interferometry
}

\author{
G. D’Amico, ${ }^{1}$ G. Rosi, ${ }^{1}$ S. Zhan, ${ }^{1, *}$ L. Cacciapuoti, ${ }^{2}$ M. Fattori, ${ }^{1}$ and G. M. Tino ${ }^{1, \dagger}$ \\ ${ }^{1}$ Dipartimento di Fisica e Astronomia and LENS, Università di Firenze, \\ INFN Sezione di Firenze, via Sansone 1, I-50019 Sesto Fiorentino (FI), Italy \\ ${ }^{2}$ European Space Agency, Keplerlaan 1, 2200 AG Noordwijk, Netherlands
}

(Received 23 May 2017; revised manuscript received 22 July 2017; published 19 December 2017)

\begin{abstract}
Gravity gradients represent a major obstacle in high-precision measurements by atom interferometry. Controlling their effects to the required stability and accuracy imposes very stringent requirements on the relative positioning of freely falling atomic clouds, as in the case of precise tests of Einstein's equivalence principle. We demonstrate a new method to exactly compensate the effects introduced by gravity gradients in a Raman-pulse atom interferometer. By shifting the frequency of the Raman lasers during the central $\pi$ pulse, it is possible to cancel the initial position- and velocity-dependent phase shift produced by gravity gradients. We apply this technique to simultaneous interferometers positioned along the vertical direction and demonstrate a new method for measuring local gravity gradients that does not require precise knowledge of the relative position between the atomic clouds. Based on this method, we also propose an improved scheme to determine the Newtonian gravitational constant $G$ towards the $10 \mathrm{ppm}$ relative uncertainty.
\end{abstract}

DOI: 10.1103/PhysRevLett.119.253201

Atom interferometers (AIs) [1] enable precise measurements of accelerations [2-5], rotations [6-9], gravity gradient (GG) [10-15], and curvature [16,17]. In most applications, optimal performances have been achieved using Ramsey-Bordé AI schemes, where the wave packets of freely falling samples of cold atoms are split and recombined using Raman or Bragg optical pulses. The excellent long-term stability and accuracy achieved so far makes them the ideal candidates for several applications, as in metrology [18-23], geodesy, geophysics, engineering prospecting, inertial navigation [24,25], and fundamental physics tests $[26,27]$. The success of this method is based on a clever mechanism that allows atoms with different initial positions and velocities to acquire the same interferometric phase in the presence of uniform forces and rotations. Large samples of cold atoms can be produced with high repetition rates and used for AI measurements with low shot noise. However, the request for AIs with higher sensitivities has revealed a limitation of this method; that is, it fails when external forces are not uniform over the size of the cloud, for instance, due to the Earth GG.

In the presence of a uniform gravity acceleration $\mathbf{g}$ and GG $\boldsymbol{\Gamma}$, the phase shift measured in an atomic gravimeter oriented along the vertical $z$ axis depends on the initial position $z_{0}$ and velocity $v_{z 0}$ of the atoms,

Published by the American Physical Society under the terms of the Creative Commons Attribution 4.0 International license. Further distribution of this work must maintain attribution to the author(s) and the published article's title, journal citation, and DOI.

$$
\phi=k_{\mathrm{eff}} g T^{2}-k_{\mathrm{eff}} \Gamma_{z z}\left(z_{0}+v_{z 0} T\right) T^{2}
$$

where $\Gamma_{z z}$ is the GG tensor component along $z, k_{\mathrm{eff}}$ is the effective wave vector of the interrogation lasers, and $T$ is the free evolution time between the central $\pi$ pulse and the $\pi / 2$ pulses acting as mirror and beam splitters of the Mach-Zehnder AI. Furthermore, the atomic wave packets at the output ports of the AI experience a non-negligible displacement, which is responsible for a loss of contrast in the AI fringes. For this reason, concerns have recently been raised on AIs for more demanding applications such as tests of Einstein's equivalence principle, gravitational waves detection, and, more generally, for metrological applications [28]. When testing the weak equivalence principle, simultaneous AIs are used to compare the free-fall acceleration of two different atomic species [29-32] or the same atom in two different quantum states [33]. Pushing the accuracy of this test to the $10^{-15}$ level in the Eötvös ratio, as proposed for future experiments on the ground [26] or in space [34], requires a control on the relative position and velocity of the two atomic samples to better than $1 \mathrm{~nm}$ and $0.3 \mathrm{~nm} / \mathrm{s}$, respectively. Such numbers are very challenging, even when using evaporatively cooled atomic samples close to the Bose-Einstein condensation phase transition, precisely positioned with optical lattice potentials. Also, in the atom interferometry measurement of the Newtonian constant of gravity performed in [21], the major source of systematic error arises from the limited control on the position and velocity of the atoms in the thermal cloud [35]. Similar limitations are affecting precise measurements of Earth's GG that are of fundamental importance for geodesy, Earth observation, and applied physics [25]. 
In this Letter, we experimentally demonstrate a new method, recently proposed in [36], to cancel the effect of the ambient GG and remove the dependency of the AI phase on the initial conditions of the atomic sample. By introducing a variation $\Delta k_{\text {eff }}$ of the effective wave vector during the central $\pi$ pulse of the Mach-Zehnder AI, it is possible to change the AI phase by $-2 \Delta k_{\text {eff }}\left(z_{0}+v_{z 0} T\right)$. When

$$
\Delta k_{\mathrm{eff}}=-\left(\Gamma_{z z} T^{2} / 2\right) k_{\mathrm{eff}},
$$

the extra phase accumulated by the atoms exactly compensates for the position- and velocity-dependent phase shift introduced by the GG [see Eq. (1)], demonstrating the possibility of operating a Ramsey-Bordé $\mathrm{AI}$ in the presence of nonuniform forces. The additional momentum $\Delta k_{\text {eff }}$ transferred to the atoms equals the momentum change due to the presence of tidal forces and it allows us to reverse the atomic trajectories in a completely symmetric way. As a consequence, the atomic trajectories perfectly overlap at the output ports of the AI [see Fig. 1(a)] without any loss of contrast. We use this method to measure GGs and curvatures. Finally, we discuss its potential for an AI determination of the Newtonian gravitational constant.
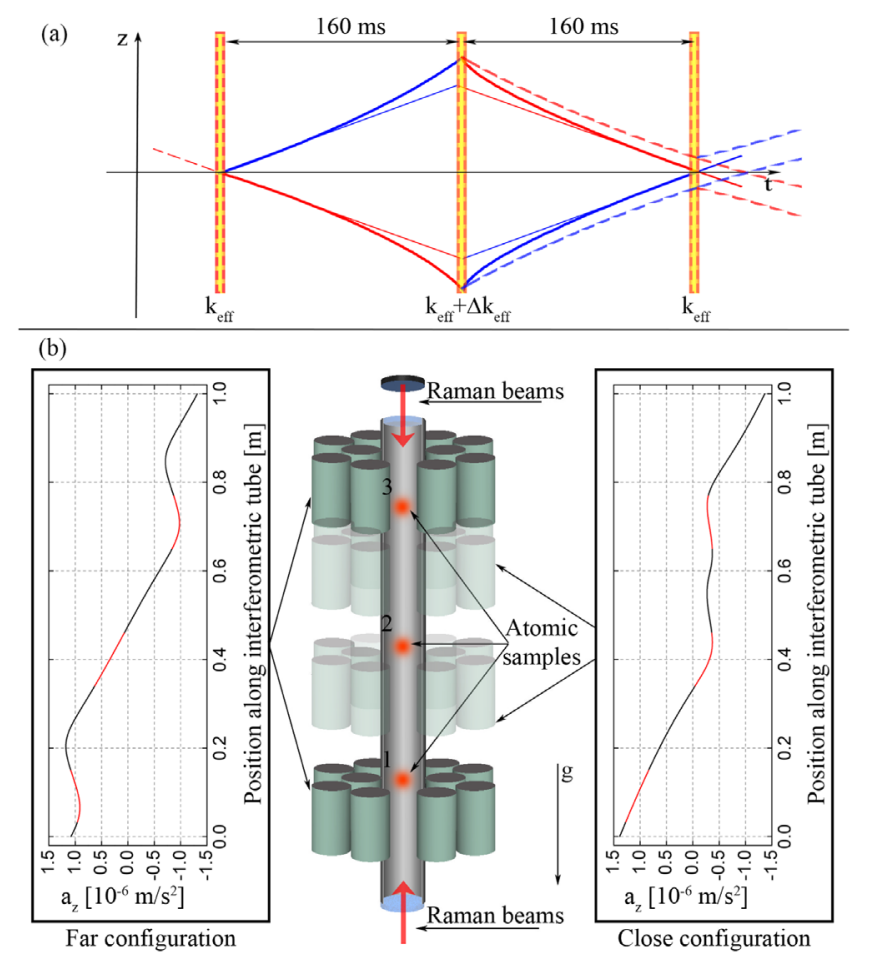

FIG. 1. (a) Space time trajectories in a freely falling MachZehnder AI operated with Raman transitions in the absence of GGs (straight thin lines) and with GGs, when the compensation scheme is not applied (dashed line) and after readapting the effective wave vector during the central $\pi$ pulse (thick curved lines). (b) Scheme of the experimental apparatus. By varying the position of the external source masses, it is possible to generate the two different vertical acceleration profiles.
A complete description of the experimental setup can be found in $[12,16]$. Here, we only discuss the measurement principle. Three clouds of ${ }^{87} \mathrm{Rb}$ atoms are magnetooptically trapped, cooled to $\sim 4 \mu \mathrm{K}$ and launched with a fixed vertical separation of $d \simeq 31 \mathrm{~cm}$ inside a 1-m-long vacuum tube. In the tube, shielded from external magnetic fields, the atomic samples are prepared in the $(F=1$, $m_{F}=0$ ) state and selected in a narrow longitudinal velocity class corresponding to a temperature of $\sim 80 \mathrm{nK}$ along the vertical direction. After state preparation, the atoms are interrogated with a $\pi / 2-\pi-\pi / 2$ sequence of vertically-oriented Raman pulses in a classical MachZehnder AI. The Raman lasers, with effective wave vector $k_{\text {eff }} \simeq 16 \times 10^{6} \mathrm{~m}^{-1}$, are resonant with the $6.8-\mathrm{GHz}$ twophoton transition between the two hyperfine levels of the ${ }^{87} \mathrm{Rb}$ ground state and have a $2-\mathrm{GHz}$ red detuning with respect to the $5^{2} S_{1 / 2}|F=2\rangle \rightarrow 5^{2} P_{3 / 2}|F=3\rangle$ transition. This detuning is changed by $\Delta \nu=c /(4 \pi) \Delta k_{\text {eff }}$ at the $\pi$ pulse of the AI to provide the required $\Delta k_{\text {eff }}$ for the compensation of the GG. The AI sequence has a duration of $2 T=320 \mathrm{~ms}$, while the entire experiment cycle takes $\sim 2 \mathrm{~s}$. The $\pi$ pulse has a duration of $24 \mu \mathrm{s}$. Interference fringes are obtained by measuring the normalized atomic population in one of the two hyperfine levels of the ${ }^{87} \mathrm{Rb}$ ground state by detecting the laser-induced fluorescence emission of the atoms. To reduce the effect of inhomogeneous magnetic fields on the AI phase, we apply the $k$-reversal protocol [37].

In our experiment, we measure the linear dependence of the gradiometer phase $\Phi$ on the frequency detuning $\Delta \nu$,

$$
\Phi(\Delta \nu)=-\left(k_{\mathrm{eff}} \Gamma_{z z} T^{2}+\frac{8 \pi}{c} \Delta \nu\right)\left(d+\Delta v_{z 0} T\right),
$$

where $\Delta v_{z 0}$ is the differential velocity between the two atomic clouds of the gravity gradiometer at the beginning of the AI sequence and $d$ is their distance. At the frequency value $\Delta \nu$ that nulls the differential phase, the two gravimeters of the gravity gradiometer show the same AI phase, independently of their position. This measurement provides a direct demonstration of the GG compensation method proposed in [36]. A linear fit to the experimental data allows us to precisely determine the frequency detuning at zero phase and from it to evaluate the average GG over the measurement baseline.

We generate different acceleration profiles by varying the vertical position of a set of high-density source masses, 24 tungsten cylinders symmetrically arranged over two platforms surrounding the AI tube (see [38] for more details). The measurements involve three simultaneous AIs that can be used to simultaneously measure three GGs, over three different baselines [see Fig. 1(b)]: gradiometer 1-2, gradiometer 2-3, and gradiometer 1-3.

In a first series of measurements, we position the source masses in the far configuration [see Fig. 1(b)]. In this way, 


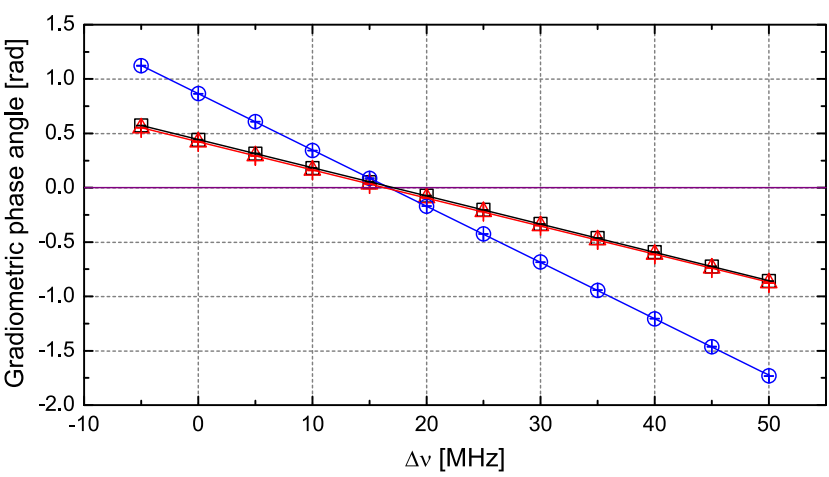

FIG. 2. Gravity gradiometer phase angle as a function of the applied $\Delta \nu$ for the three simultaneous gradiometers: results for the gradiometer 2-3,1-2, and 1-3 are in red (triangles), black (squares), and blue (circles), respectively. The source masses are positioned in the far configuration [see Fig. 1(b)].

the atoms experience a homogeneous GG on the 1-3 measurement baseline. The corresponding vertical acceleration profile is shown by the plot in the left panel, together with the regions where the three AIs take place. Each phase measurement of Fig. 2 is the result of a least-square elliptical fit of a set of 360 data points. From the three zero-crossing frequency values, it is possible to calculate the three average GGs $\Gamma_{23}=(-3.32 \pm$ $0.02) \times 10^{-6} \mathrm{~s}^{-2}, \quad \Gamma_{12}=(-3.48 \pm 0.01) \times 10^{-6} \mathrm{~s}^{-2}$, and $\Gamma_{13}=(-3.40 \pm 0.01) \times 10^{-6} \mathrm{~s}^{-2}$. The measured GG is homogeneous within $2 \%-3 \%$ over the $60-\mathrm{cm}$ baseline. As expected [see Eq. (3)], the GG measurements provided by this method do not require precise knowledge of $d$, nor do their errors change when the measurement baseline is doubled (e.g., gradiometer 1-2 and gradiometer 1-3). This technique can have interesting applications in GG measurement campaigns involving field instruments, where determining the measurement baseline $d$ with the required precision might be not straightforward.

In a second series of measurements, we position the source masses in the close configuration [see Fig. 1(b)] to produce a strong vertical variation of the GG. The vertical acceleration profile is shown by the plot in the right panel. The phase angles measured by the three gravity gradiometers as a function of the frequency detuning $\Delta \nu$ are reported in Fig. 3, together with the corresponding linear fits. In this case, $\Gamma_{23}=(0.497 \pm 0.006) \times 10^{-6} \mathrm{~s}^{-2}, \Gamma_{12}=$ $(-4.87 \pm 0.01) \times 10^{-6} \mathrm{~s}^{-2}$, and $\Gamma_{13}=(-2.193 \pm 0.006) \times$ $10^{-6} \mathrm{~s}^{-2}$. As expected, $\Gamma_{13}=\left(\Gamma_{12}+\Gamma_{23}\right) / 2$.

Interestingly, the measurement also provides the sign of the GG, without any need for applying external magnetic fields or performing additional gravity measurements. The angular coefficient of the line best fitting phase data also provides a direct measurement of the distance between the barycenters of the two clouds [39]. In our case, we obtain $d_{23}=(0.3072 \pm 0.0003) \mathrm{m}$ and $d_{12}=(0.3086 \pm 0.0004) \mathrm{m}$, both in agreement with the expected values measured

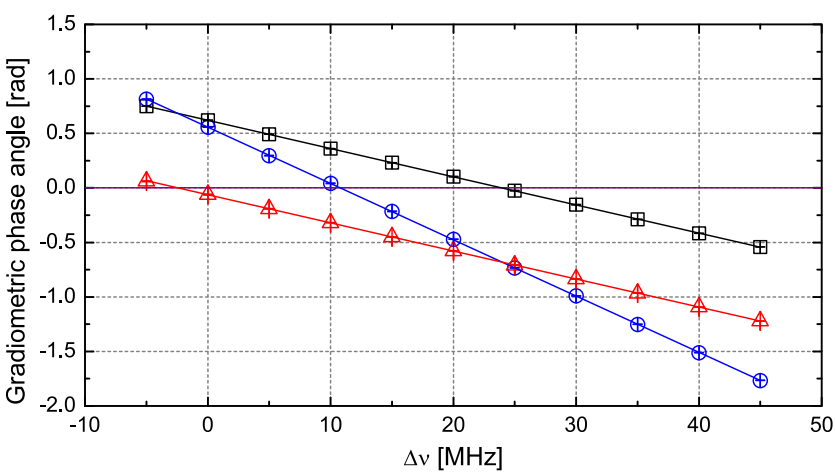

FIG. 3. As in Fig. 2, but with the source masses in the close configuration.

with the standard time-of-flight technique. Finally, we can estimate the average curvature over the measurement baseline, $\zeta=\left(\Gamma_{23}-\Gamma_{12}\right) / d=(1.743 \pm 0.004) \times 10^{-5} \mathrm{~m}^{-1} \mathrm{~s}^{-2}$.

To evaluate the stability of our instrument, we perform long-duration GG measurements with two atomic clouds separated by a distance $d$ of about $33 \mathrm{~cm}$ and with the source masses in the far configuration. The two AIs are located in the linear region of the vertical acceleration profile, where the vertical GG is highly uniform. The GG is measured with both the method of the ellipse fitting (as in $[12,16])$ and the new technique described above that, although it is still using the ellipse fitting for the determination of the gradiometer phase at different $\Delta \nu$, relies on a measurement of the zero-crossing frequency to extract the GG. The results from five measurement runs taken over five successive days are shown in Fig. 4. We observe instabilities at the level of $10 \mathrm{mrad}$, which are introduced by the measurement systematics. The major source of instability in our experiment is represented by the absolute ac Stark shift produced by the inhomogeneities in the Raman lasers intensity profile due to diffraction effects. This shift can be compensated by using the method proposed in [40]. We control the Coriolis phase shift by rotating the retroreflecting mirror with piezoactuators [41,42]. Still, even when our

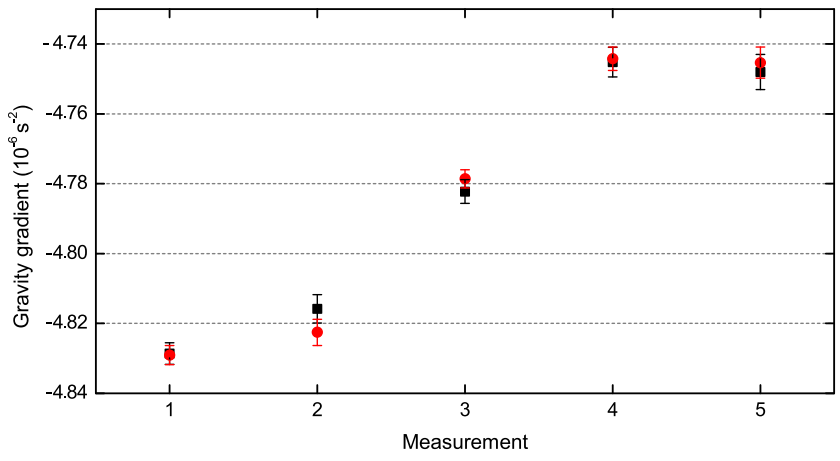

FIG. 4. GG obtained with both the method of the ellipse fitting (black circles) and the new technique based on the determination of the zero-crossing frequency (red squares) from five measurements taken over five days. Residual instabilities are due to the systematics affecting our measurements. 
compensation scheme is active, systematic shifts up to $\sim 4$ mrad can be observed for a variation of the transverse velocity of $0.1 \mathrm{~mm} / \mathrm{s}$. On the contrary, the distance between the two clouds, as measured from the slope of the linear fit to the experimental data, proves to be very stable, at the level of $300 \mu \mathrm{m}$, and reproducible from day to day. The simultaneous velocity selection pulses, which prepare the atomic clouds before starting the AI sequence, ensure that the velocity difference $\Delta v_{z 0}$ is negligible. To verify that, we have measured the gradiometer phase as a function of a small asymmetry $d T$ in the time interval between the first beam splitter pulse and the mirror pulse $(T)$ and the mirror pulse and the final beam splitter pulse $(T+d T)$. From the data, we estimate a value of $\Delta v_{z 0}$ smaller than $40 \mu \mathrm{m} / \mathrm{s}$, corresponding to a negligible correction in the measurement of $d$. Therefore, we can conclude that no contribution to the measurement systematics is coming from a drift or jitter in the relative position of the atomic clouds.

Finally, we test the robustness of the GG measurement against variations of the position of the atomic clouds. In the same configuration as before, we alternate two measurement cycles in which the position of the higher atomic sample is modulated by $\pm 1 \mathrm{~cm}$ around the $d \simeq 33 \mathrm{~cm}$ measurement baseline. Results are shown in Fig. 5. In the far configuration, we expect variations of the average GG at the level of $0.04 \% / \mathrm{cm}$ at the position of the higher cloud, therefore negligible over a distance of $2 \mathrm{~cm}$. As a consequence, GG measurements obtained with the zerocrossing frequency technique remain very stable. Relative variations are at the level of a few percent, consistent with the typical variations of the systematic shift introduced by the ac Stark effect. In contrast, measurements performed with the ellipse fitting technique show much higher relative variations. As expected, they are at the $7 \%$ level, well compatible with the relative variations $\Delta d / d$ of the measurement baseline due to the modulation of the initial position of the higher atomic cloud.

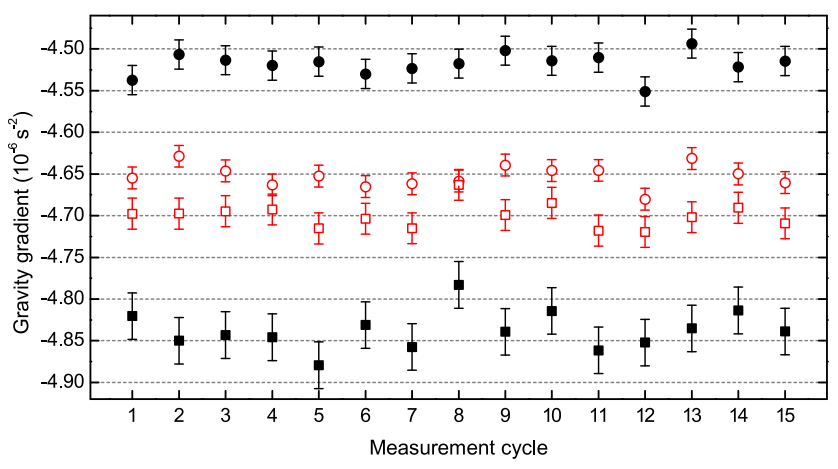

FIG. 5. GG obtained with both the method of the ellipse fitting (in black) and the new technique based on the zero-crossing frequency measurement (in red) when changing the position of the higher atomic cloud by $\pm 1 \mathrm{~cm}$ (circle and square data).
This method can be used for an improved determination of the Newtonian gravitational constant $G$. The proposed experimental setup is similar to the one described in [21]. However, rather than positioning the source masses with the purpose of generating stationary points of the gravitational acceleration, the cylinders can be uniformly distributed along the tube where atoms are interrogated with the AI sequence [43]. The resulting GG of $4.4 \times 10^{-6} \mathrm{~s}^{-2}$ is homogeneous to $2.7 \%$ over a distance of $18 \mathrm{~cm}$. Two atomic clouds at a distance of $10 \mathrm{~cm}$ can be used to probe the GG generated by the source masses in a standard MachZehnder AI with an interrogation time $T=150 \mathrm{~ms}$. The effect of Earth's GG is removed by taking the difference between measurements in two different configurations, with and without the tungsten cylinders. The GG is measured with the technique described above, which is highly insensitive to positioning errors. The gradiometer phase for different frequency detunings $\Delta \nu$ at the $\pi$ pulse of the $\mathrm{AI}$ is determined with the ellipse fitting method. Every $200 \mathrm{~s}$, a linear fit extracts the zero-crossing frequency and the corresponding GG. The frequency detuning $\Delta \nu$ is also used to open the ellipses in a controlled way and improve the fit convergence, thus avoiding the magnetic field pulses used in [21]. With this method, the relative uncertainty introduced by errors in the vertical position and size of the clouds, originally evaluated to $56 \mathrm{ppm}[21,35]$, is now reduced well below the 10-ppm level. At the same time, the smaller curvature of the gravitational acceleration in the horizontal plane also reduces the relative uncertainty due to the error on the horizontal position and size of the cloud from $44 \mathrm{ppm}$ down to $20 \mathrm{ppm}$. Performing a measurement down to the 10-ppm level requires a control on the drift in the position of the atomic clouds at the level of $100 \mu \mathrm{m} / \mathrm{s}$, which is well compatible with our setup. Alternatively, the gravity gradiometer can be operated close to the zero-crossing frequency, where compensation of the phase shift introduced by the GG can easily be ensured within $0.1 \%-1 \%$. The differential effect of the residual GG between the two configurations of the source masses is now a factor of $10^{2}-10^{3}$ less sensitive to phase instabilities and it can be measured as in [21], after introducing a magnetic field pulse to open the ellipses. In summary, the major sources of systematic error in the $G$ measurement, represented by the size of the atomic clouds and their positioning along the symmetry axis of the source masses, can easily be reduced from $72 \mathrm{ppm}$ to $20 \mathrm{ppm}$ in the current setup. By enhancing the gravity gradiometer sensitivity and improving the mass distribution, an AI determination of $G$ at the 10-ppm level or below appears feasible [43].

In conclusion, we have experimentally demonstrated the method proposed in [36] for compensating the effect of GGs in an AI, showing that a Ramsey-Bordé AI can indeed be operated to high sensitivity and accuracy in the presence of nonuniform forces. Our measurements overcome the limitations imposed by the uncertainty in the knowledge of 
initial position and velocity of the atoms contributing to the AI signal. We have used this technique for precise measurements of GGs without any need for an independent determination of the measurement baseline. Finally, we have discussed a new scheme for controlling the systematics arising from the errors on the size and the positions of the atomic clouds in a precision measurement of the Newtonian gravitational constant. Our results find important applications in different areas of research: in fundamental physics, for atom-based tests of Einstein's equivalence principle or future atom AIs for detecting gravitational waves, in metrology, for the measurement of the Newtonian gravitational constant $G$ or the fine structure constant $\alpha$, and in geodesy and Earth observation, where precision measurements of gravity, GG, and curvature are required to map the geoid or to study the mass distribution in the underground.

This work was supported by Istituto Nazionale di Fisica Nucleare (MAGIA-Advanced experiment) and Ministero dell'Istruzione, dell'Università e della Ricerca (Progetto Premiale Atom Interferometry and PRIN-2015). The authors acknowledge useful discussions with Pierre Cladè and Albert Roura.

*Also at ICPT, Trieste, Italy.

${ }^{\dagger}$ Also at CNR-IFAC, Sesto Fiorentino, Italy. guglielmo.tino@unifi.it

[1] Atom Interferometry, edited by G. M. Tino and M. A. Kasevich (SIF and IOS Press, Bologna, Amsterdam, 2014).

[2] M. Kasevich and S. Chu, Appl. Phys. B 54, 321 (1992).

[3] A. Peters, K. Y. Chung, and S. Chu, Nature (London) 400, 849 (1999).

[4] H. Müller, S. W. Chiow, S. Herrmann, S. Chu, and K. Y. Chung, Phys. Rev. Lett. 100, 031101 (2008).

[5] J. L. Gouët, T. Mehlstäubler, J. Kim, S. Merlet, A. Clairon, A. Landragin, and F. P. D. Santos, Appl. Phys. B 92, 133 (2008).

[6] T. L. Gustavson, P. Bouyer, and M. A. Kasevich, Phys. Rev. Lett. 78, 2046 (1997).

[7] T. L. Gustavson, A. Landragin, and M. A. Kasevich, Classical Quantum Gravity 17, 2385 (2000).

[8] B. Canuel, F. Leduc, D. Holleville, A. Gauguet, J. Fils, A. Virdis, A. Clairon, N. Dimarcq, C. J. Bordè, A. Landragin, and P. Bouyer, Phys. Rev. Lett. 97, 010402 (2006).

[9] A. Gauguet, B. Canuel, T. Lévèque, W. Chaibi, and A. Landragin, Phys. Rev. A 80, 063604 (2009).

[10] M. J. Snadden, J. M. McGuirk, P. Bouyer, K. G. Haritos, and M. A. Kasevich, Phys. Rev. Lett. 81, 971 (1998).

[11] J. M. McGuirk, G. T. Foster, J. B. Fixler, M. J. Snadden, and M. A. Kasevich, Phys. Rev. A 65, 033608 (2002).

[12] F. Sorrentino, Q. Bodart, L. Cacciapuoti, Y. H. Lien, M. Prevedelli, G. Rosi, L. Salvi, and G. M. Tino, Phys. Rev. A 89, 023607 (2014)

[13] X. C. Duan, M. K. Zhou, D. K. Mao, H. B. Yao, X. B. Deng, J. Luo, and Z. K. Hu, Phys. Rev. A 90, 023617 (2014).

[14] F. P. D. Santos, Phys. Rev. A 91, 063615 (2015).
[15] Y. P. Wang, J. Q. Zhong, X. Chen, R. B. Li, D. W. Li, L. Zhu, H. W. Song, J. Wang, and M. S. Zhan, Opt. Commun. 375, 34 (2016).

[16] G. Rosi, L. Cacciapuoti, F. Sorrentino, M. Menchetti, M. Prevedelli, and G. M. Tino, Phys. Rev. Lett. 114, 013001 (2015).

[17] P. Asenbaum, C. Overstreet, T. Kovachy, D. D. Brown, J. M. Hogan, and M. A. Kasevich, Phys. Rev. Lett. 118, 183602 (2017).

[18] A. Bertoldi, G. Lamporesi, L. Cacciapuoti, M. de Angelis, M. Fattori, T. Petelski, A. Peters, M. Prevedelli, J. Stuhler, and G. M. Tino, Eur. Phys. J. D 40, 271 (2006).

[19] J. B. Fixler, G. T. Foster, J. M. McGuirk, and M. A. Kasevich, Science 315, 74 (2007).

[20] G. Lamporesi, A. Bertoldi, L. Cacciapuoti, M. Prevedelli, and G. M. Tino, Phys. Rev. Lett. 100, 050801 (2008).

[21] G. Rosi, F. Sorrentino, L. Cacciapuoti, M. Prevedelli, and G. M. Tino, Nature (London) 510, 518 (2014).

[22] A. Wicht, J. M. Hensley, E. Sarajlic, and S. Chu, Phys. Scr. T102, 82 (2002).

[23] M. Cadoret, E. de Mirandes, P. Cladé, S. Guellati-Khélifa, C. Schwob, F. Nez, L. Julien, and F. Biraben, Phys. Rev. Lett. 101, 230801 (2008).

[24] A. Bresson, Y. Bidel, P. Bouyer, B. Leone, E. Murphy, and P. Silvestrin, Appl. Phys. B 84, 545 (2006).

[25] M. de Angelis, A. Bertoldi, L. Cacciapuoti, A. Giorgini, G. Lamporesi, M. Prevedelli, G. Saccorotti, F. Sorrentino, and G. M. Tino, Meas. Sci. Technol. 20, 022001 (2009).

[26] S. Dimopoulos, P. W. Graham, J. M. Hogan, and M. A. Kasevich, Phys. Rev. Lett. 98, 111102 (2007).

[27] P. Hamilton, M. Jaffe, P. Haslinger, Q. Simmons, H. Müller, and J. Khoury, Science 349, 849 (2015).

[28] A. M. Nobili, Phys. Rev. A 93, 023617 (2016).

[29] A. Bonnin, N. Zahzam, Y. Bidel, and A. Bresson, Phys. Rev. A 88, 043615 (2013).

[30] L. Zhou, S. Long, B. Tang, X. Chen, F. Gao, W. Peng, W. Duan, J. Zhong, Z. Xiong, J. Wang, Y. Zhang, and M. Zhan, Phys. Rev. Lett. 115, 013004 (2015).

[31] D. Schlippert, J. Hartwig, H. Albers, L. L. Richardson, C. Schubert, A. Roura, W. P. Schleich, W. Ertmer, and E. M. Rasel, Phys. Rev. Lett. 112, 203002 (2014).

[32] M. G. Tarallo, T. Mazzoni, N. Poli, D. V. Sutyrin, X. Zhang, and G. M. Tino, Phys. Rev. Lett. 113, 023005 (2014).

[33] G. Rosi, G. D’Amico, F. Sorrentino, M. Prevedelli, M. Zych, C. Brukner, and G. M. Tino, Nat. Commun. 8, 15529 (2017).

[34] D. N. Aguilera et al., Classical Quantum Gravity 31, 115010 (2014).

[35] M. Prevedelli, L. Cacciapuoti, G. Rosi, F. Sorrentino, and G. M. Tino, Phil. Trans. R. Soc. A 372, 20140030 (2014).

[36] A. Roura, Phys. Rev. Lett. 118, 160401 (2017).

[37] A. Louchet-Chauvet, T. Farah, Q. Bodart, A. Clairon, A. Landragin, S. Merlet, and F. P. D. Santos, New J. Phys. 13, 065025 (2011).

[38] G. Lamporesi, A. Bertoldi, A. Cecchetti, B. Duhlach, M. Fattori, A. Malengo, S. Pettorruso, M. Prevedelli, and G. M. Tino, Rev. Sci. Instrum. 78, 075109 (2007).

[39] G. W. Biedermann, X. Wu, L. Deslauriers, S. Roy, C. Mahadeswaraswamy, and M. A. Kasevich, Phys. Rev. A 91, 033629 (2015). 
[40] T. Kovachy, P. Asenbaum, C. Overstreet, C. A. Donnelly, S. M. Dickerson, A. Sugarbaker, J. M. Hogan, and M. A. Kasevich, Nature (London) 528, 530 (2015).

[41] J. M. Hogan, D. M. S. Johnson, and M. A. Kasevich, in Atom Optics and Space Physics, edited by E. Arimondo,
W. Ertmer, W. P. Schleich, and E. Rasel (IOS, Amsterdam, 2009), p. 411.

[42] S. Y. Lan, P. C. Kuan, B. Estey, P. Haslinger, and H. Müller, Phys. Rev. Lett. 108, 090402 (2012).

[43] G. Rosi, Metrologia (to be published). 